\title{
Prognostic clinical variables in childhood tuberculous meningitis: An experience from Mumbai, India
}

\author{
Sunil Karande, Vishal Gupta, Madhuri Kulkarni, Anagha Joshi* \\ Departments of Pediatrics and *Radiology, Lokmanya Tilak Municipal Medical College and General Hospital, Sion, Mumbai, India
}

\begin{abstract}
Background: In India, tuberculous meningitis (TBM) is still a major cause of neurological disabilities and death. Aim: To identify the clinical variables which predict the outcome in childhood TBM. Setting: Tertiary teaching hospital. Design: Prospective observational study. Materials and Methods: Thirty-six clinical variables were analyzed in 123 consecutive children with TBM admitted between May 2000 and August 2003. The outcome was assessed in terms of survival or death. Survival meant that the patient was discharged from hospital having made a complete recovery, or with disability. Results: Twenty-five (20\%) children recovered completely, $70(57 \%)$ survived with disability, and $28(23 \%)$ died. Employing univariate analysis nine variables correlated with survival with disability outcome: presence of tonic motor posturing, cranial nerve palsy, focal neurological deficit, hypertonia, moderate to severe hydrocephalus, cerebral infarction on cranial $\mathrm{CT}$, and requiring shunt surgery, and absence of extracranial tuberculosis and no antituberculousrelated hepatotoxicity; two variables correlated with fatal outcome: presence of deep coma (Glasgow coma scale score $<6$ ), and absence of extrapyramidal movements. When logistic regression was applied only the presence of hypertonia $(P=0.012$, d.f. $=1$, OR $0.12,95 \% \mathrm{Cl} 0.02-0.62)$ correlated with survival with disability outcome, and presence of deep coma $(P=0.030$, d.f. $=1$, OR $0.35,95 \% \mathrm{Cl} 0.14-$ $0.90)$ with fatal outcome. Conclusion: In children with TBM, the presence of hypertonia at admission is an independent predictor of neurological sequelae in survivors, and deep coma is an independent predictor of mortality.
\end{abstract}

Key Words: Hydrocephalus, meningeal tuberculosis, multivariate analysis, prognosis, computed tomography

The incidence of tuberculosis (TB) is on the increase worldwide. ${ }^{[1]}$ Between 1 and $2 \%$ of children with untreated extracranial TB develop tuberculous meningitis (TBM).$^{[2]}$ Several retrospective ${ }^{[3]-[6]}$ and prospective ${ }^{[7]-[9]}$ studies have been conducted to predict the outcome of TBM in childhood.
However, most of the data were in the pre computed tomography (CT) scan era, ${ }^{[3],[4]}$ and also the treatment protocols were not uniform or were inadequate as per current recommendations. ${ }^{[3]-[5]}$ These studies by univariate analysis identified 14 clinical variables significantly associated with a poor outcome: (i) young age, ${ }^{[3],[4],[8],[9]}$ (ii) male sex, ${ }^{[9]}$ (iii) prolonged duration of symptoms, ${ }^{[3]}$ (iv) seizures, ${ }^{[5]}$ (v) advanced stage of the disease, ${ }^{[3],[4],[6]-[9]}$ (vi) coma, ${ }^{[9]}$ (vii) tonic motor posturing, ${ }^{[9]}$ (viii) papilloedema, ${ }^{[9]}$ (ix) cranial nerve palsy ${ }_{,}{ }^{[9]}$ (x) focal neurological deficit, ${ }^{[9]}$ (xi) hydrocephalus, ${ }^{[7]}$ (xii) associated miliary TB, ${ }^{[3]}$ (xiii) absence of extra-cranial TB, ${ }^{[5]}$ and (xiv) requiring shunt surgery. ${ }^{[5]}$ Only three studies in childhood TBM[ ${ }^{[4],[5],[9]}$ have employed multivariate analysis to identify significant prognostic variables, and only one ${ }^{[9]}$ was conducted prospectively. Multivariate analysis has identified seven 'independent clinical variables' which adversely affect the outcome, viz. (i) young age ${ }^{[4],[9]}$ (ii) seizures, ${ }^{[5]}$ (iii) advanced stage of disease, ${ }^{[4],[9]}$ (iv) tonic motor posturing, ${ }^{[9]}$ (v) papilloedema, ${ }^{[9]}$ (vi) focal neurological deficit, ${ }^{[9]}$ and (vii) absence of extracranial TB. ${ }^{[5]}$

The aim of the present study was to identify the clinical variables, which predict the outcome of TBM in children.

\section{Materials and Methods}

\section{Patient enrolment}

Children with TBM, aged between 1 month and 12 years admitted to our hospital were enrolled prospectively. The study was conducted over a period of 40 months, from May 2000 to August 2003. The patient sample was by necessity a convenience sample, and all patients who met the study criteria were included in the study. All patients had an informed consent form signed by their parents.

The diagnosis of TBM was based on the clinical case definition [Table 1] devised by Doerr et al. ${ }^{[10]}$ All children had comprehensive physical and neurologic examination at the time of admission. Cerebrospinal fluid (CSF) examination and cranial CT scan were done soon after admission in every child. A standardized data entry form was used to document demographic data, clinical symptoms and signs, laboratory findings, Mantoux test result, chest radiograph and ultrasound of the abdomen findings, CSF, and cranial CT scan find- 
ings of each patient at presentation. Each patient was screened for human immunodeficiency virus (HIV) infection using the enzymelinked immunosorbent assay (ELISA) test. Pre and post-test counseling for HIV infection was offered to the parents. The ELISA kits met the minimum standards (sensitivity $>99 \%$, specificity $>$ 95\%) as recommended by the World Health Organization (WHO). ${ }^{[1]}$ The diagnosis of HIV infection was confirmed as per the WHO strategy II: when two ELISA tests based on different antigen preparations and/or different principles were positive. ${ }^{[12]}$ Family members were screened for tuberculous infection.

Nutritional status of the child was assessed by the Wellcome classification. ${ }^{[13]}$ The severity of the disease at admission was classified as per the Medical Research Council (MRC) guidelines: ${ }^{[14]}$ Stage I (early) = conscious, nonspecific symptoms, and no neurological signs; Stage II (intermediate) = signs of meningeal irritation with slight or no clouding of sensorium, with or without minor neurological deficit (cranial nerve palsy or limb paresis); Stage III (advanced) = severe clouding of sensorium, convulsions, focal neurological deficit, and/or involuntary movements. The child's level of consciousness was assessed by Glasgow coma scale (GCS). ${ }^{[15]}$ The degree and significance of hydrocephalus on the cranial CT scan was calculated by ventricular size index (VSI), a ratio of bifrontal diameter over the frontal horn diameter. ${ }^{[16]}$ A VSI of 30-38\% indicates mild hydrocephalus, $39-45 \%$ moderate hydrocephalus, and $>45 \%$ severe hydrocephalus. ${ }^{[16]}$

\section{Management of cases}

All children received standard antituberculous therapy (ATT) as recommended by the Indian Academy of Pediatrics: ${ }^{[17]}$ isoniazid ( 5 $\mathrm{mg} / \mathrm{kg} /$ day), rifampicin (10 mg/kg/day), pyrazinamide (25 mg/kg/ day), and ethambutol (20 mg/kg/day); other supportive measures (steroids, anticonvulsants, and mannitol) within 24-48 h of admission. Pyrazinamide and ethambutol were discontinued after 2 months, and isoniazid and rifampicin continued for another 4 months. Corticosteroids were administered during the first month, intravenous dexamethasone $(0.6-1.2 \mathrm{mg} / \mathrm{kg} /$ day in three divided doses $)$ for the first 7 days, followed by oral prednisolone $(2 \mathrm{mg} / \mathrm{kg} /$ day in three divided doses) and then gradually tapered over a week. Children with mild to moderate hydrocephalus received acetazolamide (20 mg/kg/ day orally in three divided doses).

All the children who had neurological deterioration during the course of the hospital stay had repeated cranial CT. Children with moderate to severe hydrocephalus and neurologic deterioration received ventriculoperitoneal (VP) shunt. ${ }^{[18]}$ When the CSF protein was more than $1 \mathrm{~g} / \mathrm{l}$, the child received initial external ventricular drainage using a chamber and later it was converted to VP shunt

Table 1: Clinical case definition of tuberculous meningitis devised by Doerr et al.. ${ }^{[10]}$

\section{Abnormal neurological signs and/or symptoms, and 2 or more} of the following

1. Discovery of adult source patient with contagious tuberculosis who had significant contact with child

2. Presence of Mantoux (5 Tuberculin units) skin test reaction $\geq 10$ $\mathrm{mm}$ of induration, or $\geq 5 \mathrm{~mm}$ of induration if child had close contact with infected adult

3. Cerebrospinal fluid abnormalities without evidence of other infectious cause

4. Abnormalities on cranial computed tomography consistent with central nervous system tuberculosis with the decrease in CSF protein. Liver function tests were done initially at a weekly interval to monitor ATT-related hepatotoxicity.

\section{Outcome assessment}

Survival or death was the outcome measure. Survival was further categorized into: (i) discharge with 'complete recovery' (no neurologic deficit), (ii) discharge with 'disability' (neurologic deficit).

\section{Data analysis}

The data were analyzed using the Statistical Package for Social Sciences, Version 11 for Windows (SPSS, Chicago, IL, USA). A univariate analysis was initially performed by the chi-square test to assess the relationship between the 36 variables and the three outcomes, viz. complete recovery, survival with disability, and death. Applying logistic regression to significant variables obtained in the univariate analysis, multivariate analysis was performed. Wherever appropriate the odds ratio (OR) was calculated and $95 \%$ confidence intervals (CI) were estimated around the OR. Statistical significance was considered as $P$ value $<0.05$. The power of analysis for the present study was $80 \%$.

\section{Results}

One hundred and twenty-three children were studied [Table $2]$. The mean age was 3.1 years (range 3 months-12 years; 土SD 2.7 years). Almost one-third of the children were malnourished: 34 had marasmus, 3 had kwashiorkor, and 1 had marasmic-kwashiorkor. Three-fourth of the children had extracranial TB: with pulmonary involvement in $87(71 \%)$, lymphadenopathy in $35(28 \%)$, and abdominal involvement in $34(28 \%)$. Forty-one (33\%) children had cranial nerve palsy: 34 had facial palsy, 20 had abducens palsy, and four had oculomotor palsy. At admission, 5 (4\%) children had Stage I disease, $12(10 \%)$ had Stage II disease, and 106 (86\%) had Stage III disease.

Cerebrospinal fluid examination was abnormal in all the children. Cellular response was lymphocytic predominance in $98(80 \%)$ children. The means (range) of CSF cell count, protein, and glucose were 254 (8-8200) cells $/ \mathrm{mm}^{3}, 1.6(0.2-8.4)$ $\mathrm{g} / \mathrm{l}$, and $0.43(0.1-1.5) \mathrm{g} / \mathrm{l}$, respectively. Admission cranial CT scan was abnormal in $121(98 \%)$ children and the hydrocephalus was the common abnormal finding in 103 (84\%) children, mild in $46(37 \%)$, moderate in $45(37 \%)$, and severe in 12 $(10 \%)$. Sixty-nine $(56 \%)$ children required shunt surgery. In three $(2 \%)$ children, ATT-induced hepatitis necessitated modified drug regimens.

Of the 123 children studied, 25 (20\%) made complete recovery ( 2 with Stage I disease, 4 with Stage II disease, and 19 with Stage III disease), $70(57 \%)$ survived with disability (3 with Stage I, 5 with Stage II, and 62 with Stage III), and 28 $(23 \%)$ died (none with Stage I, 3 in Stage II, and 25 with Stage III). The mean length of stay for children who survived to discharge was $19.8(5-90, \pm 11.7)$ days; for those who died was $20.8(3-45, \pm 17.6)$ days.

On univariate analysis, nine variables were found to be the 
predictors of good outcome, 'complete recovery:' the presence of extracranial TB $(P=0.010$, d.f. $=1)$ and ATT-induced hepatotoxicity $(P=0.043$, d.f. $=1)$; the absence of tonic posturing $(P=0.004$, d.f. $=1)$, cranial nerve palsy $(P=0.039$, d.f. $=1)$, focal neurological deficit $(P<0.001$, d.f. $=1)$, hypertonia $(P=0.006$, d.f. $=1)$, moderate-severe hydrocephalus $(P=0.004$, d.f. $=2)$, cerebral infarction $(P=0.002$, d.f. $=1)$, and shunt surgery being required $(P=0.023$, d.f. $=1$ ). Nine variables were found to be associated with 'survival with disability' outcome [Table 2]: the presence of tonic posturing $(P=0.004$, d.f. $=1)$, cranial nerve palsy $(P=$ 0.016 , d.f. $=1)$, focal neurological deficit $(P<0.001$, d.f. $=$ $1)$, hypertonia $(P=0.003$, d.f. $=1)$, moderate-severe hydrocephalus $(P=0.005$, d.f. $=2)$, cerebral infarction $(P=0.001$, d.f. $=1)$, and shunt surgery being required $(P=0.014$, d.f. $=1)$; absence of extracranial TB $(P=0.020$, d.f. $=1)$ and ATT-induced hepatotoxicity $(P=0.017$, d.f. $=1)$. Of the 19 (15\%) children without extracranial TB who survived, 18 survived with disability and only one patient made a complete recovery. Of the $93(76 \%)$ who survived without developing ATT-induced hepatotoxicity, 70 survived with disability and only 23 made a complete recovery [Table 2]. When multivariate analysis was done using logistic regression analysis [Table 3], no variable proved to be independently associated with good outcome 'complete recovery' and only the presence of hypertonia at presentation $(P=0.012$, d.f. $=1$, OR $0.12,95 \% \mathrm{CI}$ $0.02-0.62$ ) was found to be independently associated with the outcome, 'survival with disability.'

Univariate analysis identified two variables to be associated with a fatal outcome: presence of deep coma $(P=0.018$, d.f. $=1)$, and absence of extrapyramidal movements $(P=0.031$, d.f. $=1)$ at presentation. Of the $109(89 \%)$ children who did not have extrapyramidal movements at presentation, 28 died and 81 survived, of which, 23 made a complete recovery and 58 survived with disability [Table 2]. On multivariate analysis [Table 3], only the presence of deep coma $(P=0.030$, d.f.

\begin{tabular}{|c|c|c|c|c|c|c|c|c|c|c|}
\hline \multirow[t]{2}{*}{ Variables } & \multirow{2}{*}{$\begin{array}{l}\text { Number of patients } \\
(n=123) \\
n(\%)\end{array}$} & \multicolumn{3}{|c|}{$\begin{array}{l}\text { Complete recovery } \\
(n=25)\end{array}$} & \multicolumn{3}{|c|}{$\begin{array}{l}\text { Survival with disability } \\
(n=70)\end{array}$} & \multicolumn{3}{|c|}{$\begin{array}{l}\text { Death } \\
(n=28)\end{array}$} \\
\hline & & $n$ & OR & $95 \% \mathrm{Cl}$ & $n$ & OR & $95 \% \mathrm{Cl}$ & $n$ & OR & $95 \% \mathrm{Cl}$ \\
\hline \multicolumn{11}{|l|}{ Demographic variables } \\
\hline Age $<3$ years & $72(58.5)$ & 14 & 0.88 & $0.36-2.13$ & 45 & 0.71 & $0.28-1.79$ & 13 & 1.89 & $0.81-4.43$ \\
\hline Male sex & $65(52.8)$ & 15 & 1.44 & $0.59-3.52$ & 33 & 1.68 & $0.67-4.25$ & 17 & 0.66 & $0.28-1.56$ \\
\hline \multicolumn{11}{|l|}{ Symptoms } \\
\hline Illness >2 weeks & $55(44.7)$ & 14 & 1.77 & $0.73-4.29$ & 30 & 1.70 & $0.68-4.26$ & 11 & 1.33 & $0.57-3.15$ \\
\hline Fever & $109(88.6)$ & 21 & 0.60 & $0.17-2.09$ & 62 & 0.68 & $0.19-2.48$ & 26 & 0.53 & $0.11-2.53$ \\
\hline Personality changes & $74(60.2)$ & 12 & 0.54 & $0.22-1.30$ & 48 & 0.42 & $0.17-1.08$ & 14 & 1.71 & $0.73-4.01$ \\
\hline Weakness & $89(72.4)$ & 17 & 0.77 & $0.30-1.99$ & 53 & 0.68 & $0.25-1.86$ & 19 & 1.33 & $0.53-3.31$ \\
\hline Seizures & $78(63.4)$ & 16 & 1.03 & $0.41-2.58$ & 42 & 1.19 & $0.46-3.05$ & 20 & 0.63 & $0.25-1.57$ \\
\hline Contact with adult TB & $48(39.0)$ & 9 & 0.85 & $0.34-2.12$ & 24 & 1.08 & $0.42-2.80$ & 15 & 0.46 & $0.20-1.08$ \\
\hline Measles in last 6 months & $13(10.6)$ & 1 & 0.30 & $0.04-2.41$ & 9 & 0.28 & $0.03-2.35$ & 3 & 0.98 & $0.25-3.84$ \\
\hline \multicolumn{11}{|l|}{ Signs } \\
\hline BCG received & $73(59.3)$ & 17 & 1.59 & $0.63-4.04$ & 40 & 1.59 & $0.61-4.18$ & 16 & 1.13 & $0.48-2.64$ \\
\hline Malnutrition & $38(30.9)$ & 5 & 0.49 & $0.17-1.43$ & 22 & 0.55 & $0.18-1.64$ & 11 & 0.61 & $0.26-1.48$ \\
\hline $\begin{array}{l}\text { Anemia }(\mathrm{Hb}<8 \mathrm{~g} / \mathrm{dl}) \\
\text { Neurological }\end{array}$ & $19(15.4)$ & 3 & 0.70 & $0.19-2.61$ & 13 & 0.60 & $0.16-2.30$ & 3 & 1.69 & $0.45-6.27$ \\
\hline stage III of disease $†$ & $106(86.2)$ & 19 & - & - & 62 & - & - & 25 & - & - \\
\hline Deep coma & $28(22.8)$ & 4 & 0.59 & $0.18-1.88$ & 13 & 0.84 & $0.25-2.85$ & 11 & 0.34 * & $0.13-0.85$ \\
\hline Meningeal signs & $55(44.7)$ & 10 & 0.79 & $0.32-1.92$ & 32 & 0.79 & $0.31-2.00$ & 13 & 0.91 & $0.39-2.13$ \\
\hline Tonic posturing † & $26(21.1)$ & 0 & $-{ }^{*}$ & & 19 & $-{ }^{*}$ & - & 7 & 0.75 & $0.28-2.02$ \\
\hline Papilloedema & $4(3.3)$ & 1 & 1.32 & $0.13-13.25$ & 2 & 1.42 & $0.12-16.34$ & 1 & 0.88 & $0.09-8.81$ \\
\hline Optic atrophy $†$ & $6(4.9)$ & 0 & - & - & 5 & - & - & 1 & 1.50 & $0.17-13.40$ \\
\hline Cranial nerve palsy & $41(33.3)$ & 4 & 0.31 * & $0.10-0.99$ & 30 & 0.25 * & $0.08-0.82$ & 7 & 1.67 & $0.65-4.34$ \\
\hline Focal deficit & $63(51.2)$ & 5 & $0.17^{*}$ & $0.06-0.50$ & 45 & 0.14 * & $0.05-0.42$ & 13 & 1.28 & $0.55-2.98$ \\
\hline Hypertonia & $49(39.8)$ & 4 & 0.22 * & $0.07-0.70$ & 35 & 0.19 * & $0.06-0.61$ & 10 & 1.25 & $0.52-3.01$ \\
\hline Hypotonia & $44(35.8)$ & 7 & 0.64 & $0.25-1.68$ & 24 & 0.75 & $0.27-2.03$ & 13 & 0.56 & $0.24-1.32$ \\
\hline Ankle clonus & $31(25.2)$ & 7 & 1.20 & $0.45-3.22$ & 17 & 1.21 & $0.43-3.40$ & 7 & 1.01 & $0.38-2.68$ \\
\hline EP movements $†$ & $14(11.4)$ & 2 & 0.62 & $0.13-2.98$ & 12 & 0.42 & $0.09-2.03$ & 0 & $-{ }^{*}$ & - \\
\hline \multicolumn{11}{|l|}{ CSF parameters } \\
\hline Cells >100 mm³ & $54(43.9)$ & 13 & 1.51 & $0.62-3.64$ & 31 & 1.36 & $0.55-3.40$ & 10 & 1.55 & $0.65-3.71$ \\
\hline Proteins > $1 \mathrm{~g} / \mathrm{l}$ & $74(60.2)$ & 13 & 0.66 & $0.27-1.59$ & 41 & 0.77 & $0.31-1.92$ & 20 & 0.53 & $0.21-1.32$ \\
\hline \multicolumn{11}{|l|}{ CT brain parameters } \\
\hline Moderate-severe $\mathrm{HC} †$ & $57(46.3)$ & 6 & $-{ }^{*}$ & - & 40 & $-{ }^{*}$ & - & 11 & - & - \\
\hline Parenchymal enhancement & $80(65.0)$ & 16 & 0.94 & $0.38-2.36$ & 45 & 0.99 & $0.38-2.56$ & 19 & 0.85 & $0.35-2.08$ \\
\hline Basilar inflammation & $94(76.4)$ & 17 & 0.58 & $0.22-1.53$ & 55 & 0.58 & $0.21-1.60$ & 22 & 0.85 & $0.31-2.36$ \\
\hline Cerebral infarct \pm edema & $54(43.9)$ & 4 & 0.18 * & $0.06-0.57$ & 37 & 0.17 * & $0.05-0.55$ & 13 & 0.88 & $0.38-2.04$ \\
\hline Tuberculoma & $28(22.8)$ & 8 & 1.84 & $0.69-4.86$ & 13 & 2.06 & $0.73-5.80$ & 7 & 0.85 & $0.32-2.28$ \\
\hline Mantoux test positive & $20(16.3)$ & 2 & 0.39 & $0.08-1.79$ & 12 & 0.42 & $0.09-2.03$ & 6 & 0.63 & $0.22-1.84$ \\
\hline Extracranial TB & $94(76.4)$ & 24 & 9.60 * & $1.24-74.41$ & 52 & 8.31 * & $1.05-65.90$ & 18 & 2.22 & $0.88-5.59$ \\
\hline Shunt surgery required & $69(56.1)$ & 9 & 0.36 * & $0.14-0.89$ & 45 & 0.31 * & $0.12-0.81$ & 15 & 1.14 & $0.49-2.66$ \\
\hline HIV infection & $8(6.5)$ & 3 & 2.54 & $0.56-11.42$ & 4 & 2.25 & $0.47-10.85$ & 1 & 2.15 & $0.25-18.24$ \\
\hline ATT hepatotoxicity $\dagger$ & $3(2.4)$ & 2 & 8.44 * & $0.73-97.08$ & 0 & $-{ }^{*}$ & - & 1 & 0.58 & $0.05-6.65$ \\
\hline
\end{tabular}

${ }^{*} \chi^{2}$ test; $P<0.05$ significant; OR, odds ratio; $\mathrm{Cl}$, confidence interval; † OR cannot be computed. They are only computed for a $2 \times 2$ table without empty cells; ATTT, antituberculous therapy; BCG, bacilli Calmette-Guerin; CSF, cerebrospinal fluid; CT, computed tomography; EP, extrapyramidal; HC, hydrocephalus; HIV, human immunodeficiency virus; TB, tuberculosis. 
$=1$, OR $0.35,95 \%$ CI $0.14-0.90)$ proved to be independently associated with fatal outcome.

\section{Discussion}

Ours is probably the first study where clinical variables that predict complete recovery, survival with disability, and fatal outcome have been identified separately. In other studies ${ }^{[3]-[9]}$ both death and survival with moderate to severe disability were included in the poor outcome group. In the present study, $57 \%$ of the children survived with neurologic sequelae and $23 \%$ died. The reported incidence of neurologic disability in other studies ${ }^{[3]-[9]}$ varied between 32 and $56 \%$. The reported mortality ranged from 7 to $38 \%$. When compared to the other prospective study of TBM in childhood, ${ }^{[9]}$ our study had a higher number of patients (123 vs 50) and we analyzed more variables (36 vs 24).

Some of the variables found to be associated with 'survival with disability' on univariate analysis in our study, like hypertonia at the time of presentation, presence of cerebral infarction on CT, and no ATT-induced hepatotoxicity, have not been reported in the earlier studies on childhood TBM, ${ }^{[3]-[9]}$ Cerebral infarction occurs in 14-38\% of children with TBM, most commonly from the involvement of medial striate and thalamoperforating arteries. ${ }^{[19],[20]}$ This probably explains the associated disability seen in survivors. We have no proper explanation for why the absence of ATT-induced hepatotoxicity was associated with an outcome, 'survival with disability' and also absence of extrapyramidal movement disorders was associated with a fatal outcome. These aspects need detailed studies.

The results of univariate analysis do not adjust for the effect of covariates and therefore have obvious limitations. In the present study, multivariate analysis demonstrated that hypertonia at admission was the only independent predictor of the outcome 'survival with disability' and deep coma with a fatal outcome. When the power of the study was rechecked for these two significant variables, it was $76.4 \%$ for hypertonia as a predictor of the outcome 'survival with disability' and $86.9 \%$ for deep coma as predictor of fatal outcome. These precise findings have not been reported in earlier studies. ${ }^{[4],[5],[9]}$

The strengths of the present study are that: (i) it was a prospective study including 123 children, (ii) patient population received uniform treatment protocols, and (iii) a large number of variables were analyzed for significance. However, the present study has its limitations. First, the diagnosis of TBM was based on clinical criteria and not on microbiological confirmation. Although definitive diagnosis of TBM depends

\begin{tabular}{|c|c|c|c|c|c|c|}
\hline Variables & $\begin{array}{l}\text { Regression } \\
\text { coefficient }\end{array}$ & SE & $P$ value * & d.f. & OR & $95 \% \mathrm{Cl}$ \\
\hline \multicolumn{7}{|l|}{ Complete recovery } \\
\hline Hypertonia & -1.41 & 0.75 & 0.061 & 1 & 0.24 & $0.06-1.07$ \\
\hline Extracranial TB & 1.75 & 1.14 & 0.124 & 1 & 5.76 & $0.62-53.59$ \\
\hline Focal deficit & -1.04 & 0.71 & 0.143 & 1 & 0.36 & $0.09-1.42$ \\
\hline Cerebral infarct \pm edema & -0.98 & 0.71 & 0.171 & 1 & 0.38 & $0.09-1.52$ \\
\hline Hydrocephalus & - & - & 0.178 & 2 & - & - \\
\hline Mild & -1.93 & 1.04 & 0.063 & 1 & 0.15 & $0.02-1.11$ \\
\hline Moderate-severe & -0.84 & 0.75 & 0.265 & 1 & 0.43 & $0.10-1.89$ \\
\hline ATT hepatotoxicity & 2.63 & 2.48 & 0.288 & 1 & 13.87 & $0.11-17.75 E+2$ \\
\hline Cranial nerve palsy & -0.31 & 0.81 & 0.700 & 1 & 0.73 & $0.15-3.57$ \\
\hline Tonic posturing & -7.81 & 28.84 & 0.787 & 1 & 0.00 & $0.00-14.31 E+20$ \\
\hline Shunt surgery required & -0.13 & 0.77 & 0.867 & 1 & 0.88 & $0.20-3.96$ \\
\hline Constant & 18.02 & 57.95 & 0.756 & 1 & $66.93 \mathrm{E}+6$ & - \\
\hline \multicolumn{7}{|l|}{ Survival with disability } \\
\hline Hypertonia & -2.13 & 0.85 & 0.012 & 1 & 0.12 & $0.02-0.62$ \\
\hline Focal deficit & -1.30 & 0.80 & 0.103 & 1 & 0.27 & $0.06-1.30$ \\
\hline Hydrocephalus & - & - & 0.152 & 2 & - & - \\
\hline Mild & 0.45 & 1.00 & 0.654 & 1 & 1.56 & $0.22-11.02$ \\
\hline Moderate-severe & 1.86 & 1.11 & 0.095 & 1 & 6.42 & $0.72-57.02$ \\
\hline Extracranial TB & 1.72 & 1.26 & 0.172 & 1 & 5.56 & $0.48-64.98$ \\
\hline Cerebral infarct \pm edema & -0.91 & 0.73 & 0.213 & 1 & 0.40 & $0.10-1.69$ \\
\hline Cranial nerve palsy & -0.64 & 0.91 & 0.482 & 1 & 0.53 & $0.09-3.15$ \\
\hline Tonic posturing & -7.88 & 32.98 & 0.811 & 1 & 0.00 & $0.00-44.47 E+23$ \\
\hline Shunt surgery required & -0.18 & 0.83 & 0.826 & 1 & 0.83 & $0.17-4.21$ \\
\hline ATT hepatotoxicity & 9.77 & 112.05 & 0.931 & 1 & $17.52 \mathrm{E}+3$ & $0.00-4.21 E+99$ \\
\hline Constant & 2.87 & 233.62 & 0.990 & 1 & 17.55 & - \\
\hline \multicolumn{7}{|l|}{ Death } \\
\hline Deep coma & -1.05 & 0.48 & 0.030 & 1 & 0.35 & $0.14-0.90$ \\
\hline EP movements & 8.06 & 26.29 & 0.759 & 1 & $31.49 \mathrm{E}+2$ & $0.00-75.00 E+24$ \\
\hline Constant & -15.37 & 52.58 & 0.770 & 1 & 0.00 & - \\
\hline
\end{tabular}

${ }^{\star} P<0.05$ significant; SE, standard error; d.f., degree of freedom; OR, odds ratio; CI, confidence interval; ATT, antituberculous therapy; EP, extrapyramidal; TB, tuberculosis 
on the detection of the tubercle bacilli in the CSF, either by smear examination or by bacterial culture; smears are usually positive in fewer than $10 \%$ of cases of TBM, while culture for Mycobacterium tuberculosis takes up to 8 weeks and also often negative. ${ }^{[18],[21]}$ Most studies on outcome in childhood TBM have similarly diagnosed patients predominantly on clinical criteria..$^{[3]-[7],[9]}$ Second, the neurological outcome in children who survived was assessed purely on clinical examination at the time of discharge. We did not perform formal intelligence quotient or audiological evaluations. Third, since follow up after discharge from hospital is generally poor in our setting, we could not document the outcome after completion of ATT.

In conclusion, the present study documents that TBM continues to be a serious childhood illness with a high neurologic morbidity and mortality. We report that in children with TBM on admission to hospital the presence of hypertonia is an independent predictor of neurological sequelae in survivors, and the presence of deep coma is an independent predictor of mortality. Additional prospective studies employing multivariate analysis are required to determine the generalization of our findings.

\section{Acknowledgments}

We thank our Dean, Dr. M. E. Yeolekar, for granting us permission to publish the hospital data; our Biostatistician, Mr. Kailas Gandewar, and Dr. D.P. Singh, Reader, Department of Research Methodology, Tata Institute of Social Sciences, Deonar, Mumbai for their help in the statistical analysis of the data.

\section{References}

1. Dye C, Scheele S, Dolin P, Pathania V, Raviglione MC. Consensus statement Global burden of tuberculosis: estimated incidence, prevalence, and mortality by country. WHO Global Surveillance and Monitoring Project. JAMA $1999 \cdot 282 \cdot 677-86$

2. Starke JR. Tuberculosis of the central nervous system in children. Semin Pediatr
Neurol 1999;6:318-31.

3. Delage G, Dusseault M. Tuberculous meningitis in children: a retrospective study of 79 patients, with an analysis of prognostic factors. Can Med Assoc J 1979;120:305-9.

4. Humphries MJ, Teoh R, Lau J, Gabriel M. Factors of prognostic significance in Chinese children with tuberculous meningitis. Tubercle 1990;71:161-8.

5. Paganini H, Gonzalez F, Santander C, Casimir L, Berberian G, Rosanova MT Tuberculous meningitis in children: clinical features and outcome in 40 cases. Scand J Infect Dis 2000;32:41-5.

6. Lee LV. Neurotuberculosis among Filipino children: an 11 years experience at the Philippine Children's Medical Center. Brain Dev 2000;22:469-74.

7. Altunbasak S, Alhan E, Baytok V, Aksaray N, Yuksel B, Onenli N. Tuberculous meningitis in children. Acta Paediatr .Jpn 1994;36:480-4

8. Thilothammal N, Krishnamurthy PV, Banu K, Ratnam SR. Tuberculous meningitis in children - clinical profile, mortality and morbidity of bacteriologically confirmed cases. Indian Pediatr 1995;32:641-7.

9. Mahadevan B, Mahadevan S, Serane VT. Prognostic factors in childhood tuberculous meningitis. J Trop Pediatr 2002;48:362-5.

10. Doerr CA, Starke JR, Ong LT. Clinical and public health aspects of tuberculous meningitis in children. J Pediatr 1995;127:27-33.

11. Joint United Nations Programme on HIV/AIDS (UNAIDS) - World Health Organization. Revised recommendations for the selection and use of HIV antibody tests. Wkly Epidemiol Rec 1997;72:81-8.

12. Dodd CL, Greenspan D, Katz MH, Westenhouse JL, Feigal DW, Greenspan JS. Oral eandidiasis in HIV infection: pseudomembranous and erythematous candidiasis show similar rates of progression to AIDS. AIDS 1991;5:1339-43.

13. Welleome Trust Working Party. Classification of infantile malnutrition. Lancet 1970;ii:302-3

14. Medical Research Council. Streptomycin in Tuberculosis Trials Committee. Streptomycin treatment of tuberculous meningitis. Lancet 1948;i:582-96.

15. Teasdale GM, Jennett B. Assessment and prognosis of coma after head injury Acta Neurochir (Wien) 1976;34:45-55.

16. TerBrugge KG, Rao KC, Lee SH. Hydrocephalus and atrophy. In: Lee SH, Rao KC, editors. Cranial Computed Tomography and MRI. 2nd ed. New York: McGraw Hill; 1987. pp231-62.

17. Indian Academy of Pediatrics. Treatment of childhood tuberculosis: Consensus Statement of IAP Working Group. Indian Pediatr 1997;34:1093-6.

18. Newton RW. Tuberculous meningitis. Arch Dis Child 1994;70:364-6.

19. Ozates M, Kemaloglu S, Gurkan F, Ozkan U, Hosoglu S, Simsek MM. CT of the brain in tuberculous meningitis. A review of 289 patients. Acta Radiol $2000 ; 41: 13-7$.

20. Hsieh FY, Chia LG, Shen WC. Locations of cerebral infaretions in tuberculous meningitis. Neuroradiology 1992;34:197-9.

21. Kumar R, Singh SN, Kohli N. A diagnostic rule for tuberculous meningitis Arch Dis Child 1999;81:221-4.

Accepted on 06-03-2005

\section{Invited Comments}

In developing countries, childhood tuberculous meningitis (TBM) remains an important cause of neurological handicap and death. Current antituberculosis treatment is remarkably effective if administered timely; unfortunately, as in the present study, the diagnosis of childhood TBM is often delayed, resulting in severe neurological sequelae and even death.

Early diagnosis of TBM is notoriously difficult; the symptoms in stage 1 TBM are subtle, nonspecific, and relate more to the underlying primary infection (lung tuberculosis) than to the brain. Frequent diagnoses include gastroenteritis, otitis media, or upper respiratory infection. Poor weight gain, or loss of weight, reflected by crossing of weight centiles on the Road to Health Card, is an extremely valuable clue to early diagnosis. ${ }^{[1]}$ In high prevalence tuberculosis areas, this should alert the clinician to the possibility of tuberculosis. A positive contact history, a chest radiograph and tuberculin skin testing are other most valuable simple procedures that may support an early tuberculosis diagnosis. ${ }^{[2]}$

Unfortunately, however, patients are often repeatedly seen without TBM being considered, resulting in disease progression to stages 2 and 3 TBM. ${ }^{[3]}$ The majority of these patients will now have evidence of obstructive hydrocephalus on cranial computerized tomography (CT). Although noncommunicating hydrocephalus can result in serious neurological handicap and even sudden death due to cerebral herniation, if not recognized and managed appropriately, most 
cases of childhood tuberculous hydrocephalus are communicating and respond well to medical treatment (antituberculosis drugs and diuretics). Progressive tuberculous periarteritis, however, almost invariably results in infarction of the basal ganglia, the clinical correlates of which are focal abnormalities of power, tone, and/or abnormal movements. Brainstem microinfarcts, although rarely demonstrated by CT, are a common autopsy finding in advanced TBM and present clinically with deep coma and decerebration.

The present study prospectively assessed an impressive array of clinical features in a large cohort of childhood TBM patients with regard to the clinical outcome. These features were separately evaluated for neurological handicap and death by both univariate and multivariate analyses. A significant number of variables correlated independently with these outcomes with regard to prognosis. However, on multivariate analysis only hypertonia featured as a prognostic indicator for neurological handicap and only deep coma for death. We agree with the authors that these findings most likely relate to vasculitis and infarction since most patients in this study had stage 3 TBM.

The results of this study re-emphasize the need for early diagnosis of this devastating disease.

Johan Schoeman, Peter Donald

Department of Paediatrics and Child Health, Faculty of Medical Sciences, University of Stellenbosch, Cape Town, South Africa. E-mail: jfs@sun.ac.za

\section{References}

1. Donald PR, Schoeman .JF, van Schalkwyk H.JS. The "Road to Health Card" in Tuberculous Meningitis. J Trop Pediatr 1985; 31: 117-120.

2. Donald PR, Schoeman JF, Cotton MF, van Zyl LE, Strachan G. Missed opportunities for the prevention and early diagnosis of tuberculous meningitis in children. S Afr J Epidemiol Infect 1990; 5: 76-78.

3. Nuver .J, Vloedbeld M, Schoeman .JF Gerritsen .J, van der Palen .J, van der Werf TS, Hoekstra MO. Factoren van belang voor vroege diagnose van tuberculeuze meningitis. Tijdschr Kindergeneesk 2001; 69: 9-13. [abstract in English] 